\title{
Pain management in acute otitis media: a qualitative study of parents' views and expectations
}

\author{
Rick T. van Uum ${ }^{1 *} \mathbb{D}$, Roderick P. Venekamp ${ }^{1}$, Anne G. M. Schilder ${ }^{1,2}$, Roger A. M. J. Damoiseaux ${ }^{1}$ and \\ Sibyl Anthierens ${ }^{3}$
}

\begin{abstract}
Background: For unclarified reasons, parents tend to be cautious about administering analgesics to their children, potentially leading to suboptimal management of AOM symptoms. We aim to understand parents' views and expectations of pain management in acute otitis media (AOM) in children.

Methods: Qualitative study alongside a cluster-randomised controlled trial (PIM-POM study) aimed at optimising pain management in childhood AOM. We purposefully sampled 14 parents of children diagnosed with AOM by their GP, who were recruited to the trial between November 2017 and May 2018. Semi-structured interviews were held at home in the first two weeks after trial enrollment. Interviews were audio-recorded, transcribed and analyzed thematically.

Results: Parents experienced difficulties in recognising earache and other symptoms of an ear infection. They consulted the GP for a diagnosis, for reassurance and for management advice. Parents shared that, prior to consultation, they had insufficient knowledge of the benefits of correctly dosed pain medication at regularly scheduled intervals. Parents valued the GP's advice on pain management, and were happy to accept pain medication as standalone therapy, provided that the GP explained why antibiotics would not be needed. Parents' views and expectations of pain management in AOM were shaped by previous experiences of AOM within their family; those with a positive experience of pain medication are more likely to use it in subsequent AOM episodes.
\end{abstract}

Conclusions: Parents of children with AOM consult the GP to help cope with uncertainties in recognising symptoms of AOM, and to receive management advice. It is important that GPs are aware of parents' lack of understanding of the role of pain medication in managing AOM, and that they address this during the consultation.

Trial registration: Netherlands Trial Register, identifier NTR4920 (registration date: 19 December 2014).

Keywords: Acute otitis media, Children, Parents, Pain management, Primary care, Qualitative study

\section{Background}

Acute otitis media (AOM) is among the most common infectious diseases and reasons for doctor consultations and antibiotic prescribing in children [1-3]. Earache is central to children's and parents' experience of the condition $[4,5]$, Although it is widely accepted that this is best managed with analgesics that are dosed based upon weight $[6,7]$; its best use is often mentioned casually

\footnotetext{
* Correspondence: R.T.vanUum-2@umcutrecht.nl

${ }^{1}$ Julius Center for Health Sciences and Primary Care, University Medical Center Utrecht, University Utrecht, Office number FAC 5.09, P.O. Box 85500, 3508, GA, Utrecht, The Netherlands

Full list of author information is available at the end of the article
}

rather than discussed specifically during consultations for AOM [8, 9], Importantly, parents tend to be cautious about administering analgesics to their children, due to concerns about adverse effects or a lack of clear instructions $[10,11]$, This may result in suboptimal management of AOM symptoms, in particular earache, as well as future reconsultation and antibiotic prescriptions.

Surprisingly little research has been done to understand parents' views and expectations of pain management for $\mathrm{AOM}$ in children. Most strategies to improve AOM management so far have focused on reducing antibiotic prescriptions, targeting medical professionals [12, 13], thereby overlooking parents' key role in decision making.

(c) The Author(s). 2019 Open Access This article is distributed under the terms of the Creative Commons Attribution 4.0 International License (http://creativecommons.org/licenses/by/4.0/), which permits unrestricted use, distribution, and reproduction in any medium, provided you give appropriate credit to the original author(s) and the source, provide a link to the Creative Commons license, and indicate if changes were made. The Creative Commons Public Domain Dedication waiver (http://creativecommons.org/publicdomain/zero/1.0/) applies to the data made available in this article, unless otherwise stated. 
We therefore conducted a qualitative study alongside a trial that aimed to optimise pain management in children with AOM.

\section{Methods}

In this process evaluation, we interviewed parents of children who participated in the trial (PIM-POM study). The trial was a primary care-based, cluster-randomised controlled trial of a multifaceted educational intervention that aimed to optimise pain management in children with GP-confirmed AOM and ear pain. In this trial, 37 Dutch GP practices were randomly allocated to either the intervention or control group. Prior to the first inclusion, GPs in the intervention group received an educational intervention consisting of an online training module and a face-to-face visit by the study physician. Both interventions aimed at educating GPs about pain management in AOM; encouraging them to use a parent information leaflet to discuss pain management in each AOM consultation and prescribe both paracetamol and ibuprofen according to current guidelines [14], rather than rely on over-the-counter (OTC) purchase of these medications. GPs in the control group did not receive this educational intervention and provided usual care. To ensure standardised AOM diagnosis, all participating GPs, in both intervention and control group, completed an online module illustrating various otoscopic images (i.e. normal appearance of tympanic membrane, AOM, and otitis media with effusion). The main outcome measure of the trial was the mean difference in ear pain score on Wong-Baker Faces Scale over the first three days [15]. Full details on the trial's rationale and design have been published elsewhere [16]. The translated parent information leaflet is available in Additional file 1.

\section{Study population and setting}

We asked parents of children, recruited to the trial between November 2017 and May 2018, to take part in this interview study. They were approached by phone within three days of enrollment. We purposefully sampled parents from both the intervention and control group, taking into account parents' age, sex, education level, and employment status. We expected to conduct 10 to 20 semi-structured interviews to reach data sufficiency [5] [17].

\section{Data collection and analysis}

After obtaining written informed consent, the study physician $(\mathrm{RTVU})$ interviewed parents at their homes within two weeks of trial enrollment. Interviews were semi-structured, and focused on the management of their child's current AOM episode, in particular pain management. The interview guide (see Table 1 and Additional file 1) was constructed through literature review and input of the expert
Table 1 Summary of the interview guide ${ }^{a}$

Topics

What are your views on earaches?

How can you tell when your child has an earache?

What are your greatest concerns?

What do you do when your child has an earache?

What reasons made you contact the GP (practice)?

What did the GP discuss with you?

Which aid did the GP use to support what he/she was telling you?

Did the GP change your previously-held ideas/opinions about OMA and its treatment?

How did the recent appointment differ from your previous visit(s)?

Did the prescribed pain medication help?

Has your participation in the study impacted your views on OMA and pain relief?

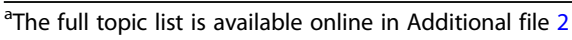

research team. All interviews were audio-recorded and transcribed verbatim. Interviews were analyzed thematically in an iterative process $[18,19]$.

$\mathrm{R}, \mathrm{TvU}$ and SA (sociologist) reviewed the initial interview transcripts for accuracy and decided whether the interview guide needed adaptation. The same researchers analyzed and coded the first four interviews, line by line. Next, the open codes were grouped into bigger codes; categories with common meaning were grouped. Descriptions of each theme and sub-theme were added, along with quotes to support each. In order to ensure the clarity of the themes $R \operatorname{TvU}, \mathrm{SA}, \mathrm{RV}, \mathrm{RD}$ discussed and interpreted these categories into potential themes. These themes were discussed within the team and reformulated at several stages during the analysis process. We collected data until saturation was reached.

\section{Results}

Between November 2017 to May 2018, 18 GP practices across the Netherlands recruited 44 children with AOM to the PIM-POM study. We invited parents of 19 of these children to take part in this qualitative study; 25 parents were not actively approached for various reasons: not being able to reach parents by phone, insufficient time during phone call to obtain proper informed consent, or due to purposeful sampling (parents with similar characteristics already present in study sample). Of the invited parents, six parents declined due to time constraints and 14 parents of 13 children consented to be interviewed (mean interview duration was $19 \mathrm{~min}$ ).

Table 2 summarises the parents' characteristics; nine mothers and five fathers, with a range of educational backgrounds and employment status. Most parents had one or two children, had consulted the GP up to two times for their child's current AOM episode, and half of the children attended daycare. 
Table 2 Baseline characteristics

\begin{tabular}{lllll}
\hline No & Gender & Age & Trial group & Education \\
\hline 1 & Female & $35-40$ & Control & Higher professional \\
2 & Female & $30-35$ & Intervention & Higher professional \\
3 & Male & $30-35$ & Intervention & University \\
4 & Male & $30-35$ & Intervention & Higher professional \\
5 & Female ${ }^{a}$ & $25-30$ & Intervention & Vocational training \\
6 & Male & $30-35$ & & Vocational training \\
7 & Female & $35-40$ & Control & Vocational training \\
8 & Female & $40-45$ & Control & Higher professional \\
9 & Female & $35-40$ & Control & Higher professional \\
10 & Male & $30-35$ & Control & University \\
11 & Female & $30-35$ & Intervention & Vocational training \\
12 & Female & $25-30$ & Intervention & None \\
13 & Male & $40-45$ & Intervention & Vocational training \\
14 & Female & $30-35$ & Intervention & University \\
\hline
\end{tabular}

Parents of the same child

Three themes developed from the analysis: reasons for consultation, empowerment of parents in managing $\mathrm{AOM}$, and future AOM management.

\section{Reasons for consultation \\ Recognising AOM}

Parents found it difficult to recognise symptoms of AOM in their child, in particular earache, and distinguishing AOM symptoms from those of other common childhood conditions, such as teething or the common cold. Some parents felt guilty for not recognising symptoms of AOM early on as they felt that their child suffered considerably.

R2: "...and the trouble is that it takes a few days before I realise we are dealing with an ear infection. So by then, he [child] has been cranky for some time; before he develops an obvious fever and can express that he is in pain, we are at least two days on."

Parents' awareness of their child's suffering was shaped by their own experience of earache; parents who remembered how they themselves had suffered from earache as a child chose to seek medical care for their child urgently.

\section{R2: I have had AOM episodes as a child, I vividly recall them, each slight breeze of wind in my ear was as if someone stuck a knife in my ear [makes a stabbing gesture]. That is why I feel we should immediately act on it, together with the GP.}

Parents consulted the GP to receive a diagnosis, as they found it difficult to recognise $\mathrm{AOM}$ as the underlying cause of their child's symptoms. Consultation was triggered by a range of symptoms, in particular fever, earache, sleep disturbance, general illness, and excessive crying. In other instances, parents consulted when they felt self-managing the illness had failed.

\section{Reassurance}

A comprehensive history and physical examination, in particular otoscopic examination, made parents' feel that they were taken seriously and that they received the correct diagnosis and treatment.

R9: "...that, whether she [GP] confirms it [ear infection] or not, at least she will have a look in his ears. That she takes a moment to look at her [child], just to see what is going on, so that I know what to do and expect. Because, obviously, I cannot say whether she has an ear infection or not. It is just as likely she has a tooth coming in, and... that certainty, really, is what I am after..."

Apart from receiving a diagnosis, parents consult their GP to rule out severe illnesses or complications; they would rather consult 'unnecessarily', than fail to recognise a severe illness.

R2: "...I feel like it is better to go [to the GP] ten times, and be told there is nothing wrong, or only something minor...than, uh, to have waited too long even once...".

\section{Management advice}

Parents sought advice on how to best manage their child's symptoms, and felt their GP was the best person to give this information. They mostly wanted practical advice that provides rapid symptom relief.

R7: ... to be certain she actually has an ear infection, and to see whether it can be treated, to relieve her earache.

\section{Empowerment of parents in managing AOM Advice on analgesics in general}

Many parents were unaware that AOM is usually self-limiting, neither were they aware of the importance and the potential benefits of pain medication, and how to best give these to their child.

R2: "I am always cautious with pain medication and he [GP] really explained that - that there is no need for that [...] I did not know that pain could have such a big impact on his [child] day, you know, and that you could relieve it so effectively with paracetamol."

Once parents felt they recognised earache or an ear infection, most initially self-managed this with treatments, 
such as olive oil ear drops, nasal saline or decongestant sprays, and paracetamol.

Many parents were cautious about giving paracetamol in the recommended dose and frequency; they would only give paracetamol when they felt it was absolutely necessary, and often dosed conservatively.

Parents who had received information from their GP about weight-based dosed paracetamol at regularly scheduled intervals (regardless of trial group) often had not realised, prior to consultation, that this regimen is safe and more effective. They appreciated advice on pain management and were happy to accept pain medication as standalone therapy, provided that their GP had performed a complete physical examination of their child, and had explained the natural course of AOM and why antibiotics were not needed.

\section{R2: "Well, I used to have the idea that pain} medication was not enough, which is why, if things went on too long, I would ask for antibiotics on top of that. And now I see that pain medication alone is fine, that you still see improvement with only that."

Parents who had received information on weight-based dosing during the consultation expressed that they closely adhered to the GP's recommendations afterwards.

R3: "yes, we simply did provide, at first the maximum dosage and then after two days we adjusted to half the dosage [as was advised in the leaflet]."

\section{Advice on ibuprofen}

In those cases where the GP advised parents to give their child ibuprofen in addition to paracetamol, it was revealed that parents were not aware that it was safe to give ibuprofen to children prior to the GP's advice. Neither did they know about the OTC availability of ibuprofen. Parents attributed stronger analgesic properties to ibuprofen than to paracetamol. Those who gave ibuprofen to their children felt it was effective.

R4: “...it [ibuprofen] worked extremely well. I felt it worked better than paracetamol, actually... I really thought so; with it, she [patient] was able to feel better for a few hours. And before, with paracetamol, we [parents] did things differently, too, because we were hesitant to give it during the day. It was more something for night-time, when she needed to sleep..."

\section{Discussing antibiotics}

Parents were cautious about antibiotics, especially when prescribed repeatedly. When asked, parents referred to potential harmful effects of (repeated) antibiotic use such as antibiotic resistance. Participants felt that GPs rarely discuss the potential benefits and harms of antibiotics for AOM. Parents were happy to refrain from antibiotics when their GP carefully explained why antibiotics were unnecessary.

R4: "...that it is true, you know, they do not always need antibiotics to get better. In the past, that had often been the case with her [child] ..."

\section{Supportive material}

In general, parents felt that supportive material such as information leaflets, booklets or medical websites is useful. Parents in the intervention group valued the trial's information leaflet and felt that it provided clear instructions on dosage and frequency of paracetamol and ibuprofen. The leaflet allowed them to recap the information at home. Parents valued GP's recommendations regarding visiting websites that provide trusted information. Parents expressed having more faith in the information provided by their GPs than collected by themselves from other sources.

R8: "Well, if you are familiar with it [ear infection], that means you have already Googled it, so I... but when I come home and I do not know exactly what is wrong with her [child], yeah, if you Google 'earache,' well...you read some pretty shocking things. So then it is nice when he [GP] comes right out and says, go ahead and look it up...".

\section{Prescriptions versus OTC use of analgesics}

Parents (regardless of trial group) felt there was no need for a prescription of paracetamol or ibuprofen since these drugs are widely available OTC. They considered GPs' instructions, together with those given by the pharmacist, sufficient. Certain parents, however, felt that a prescription for ibuprofen may, in general, lower the threshold for (other) parents to actually give this drug.

\section{Future AOM management}

Parents who had a positive experience with pain medication had developed a greater self-efficacy in regards to self-management of their child's AOM. They were more inclined to give these drugs in the appropriate dose if symptoms would recur. GP advice on the use of pain medication enhanced parents' confidence in this particular management strategy, and made them more willing to initiate pain medication in subsequent episodes. Furthermore, they felt more confident to give higher and more regular doses of pain medication. Parents also 
reported that GP's advice on ibuprofen had lowered their threshold to use this in the future.

\section{R12: "...I think that I would then [with subsequent ear infection] be more likely to stick to pain medication and try to get by without using antibiotics..."}

Nevertheless, some parents did not feel confident to manage future AOM episodes themselves and would re-consult their GP in the future to rule out potential underlying or serious illness that can only be identified by clinicians. This is particularly the case in situations where parents were previously faced with a severe illness in their child or when they had older children with similar or recurrent problems.

\section{R2: "I would probably handle it [future AOM episode] exactly as I did this time. So, first I would see the GP to find out if one or both ears are involved. And after that, yeah, try to get by on pain medication as much as possible."}

\section{Discussion}

\section{Main findings}

Parents of children with AOM seek professional help to receive a diagnosis, reassurance and management advice. Parents value GP's advice on the use of pain medication; they generally have insufficient knowledge of the role of pain medication in AOM. They gained new knowledge and accept pain medication as standalone therapy, provided that the GP explains why antibiotics would not be needed. Parents' views and expectations of pain management in AOM are shaped by previous experiences of AOM within their family; those with a positive experience of pain medication are more likely to use it in subsequent episodes. Parents valued consultations that provided management advice on pain medication and reassurance when consulting for $\mathrm{AOM}$, especially when antibiotics were not needed according to the GP.

\section{Comparison with existing literature}

Few studies so far have explored parents' views of AOM management, and these primarily focussed on the role of antibiotics [5] [20-22], Among a convenience sample of parents of children attending Australian day-care centres, Hansen et al. found that most parents assume that antibiotics were the best treatment option [5]. These parents were primarily higher-educated mothers. They all gave their children pain medication for temperature reduction or pain relief, but they considered these drugs alone insufficient to treat AOM. Meherali et al. found the same in purposive and convenience samples from a paediatric emergency department in Canada [22].
In contrast, we found that parents do accept pain medication as standalone treatment, when the GP has taken time to discuss why antibiotics are not needed. We also found that Dutch parents are cautious about using antibiotics, and would rather avoid them if possible. In the Netherlands, a watchful waiting strategy for AOM has been advocated for decades, and antibiotic prescription rates are among the lowest globally. Nevertheless, our findings are consistent with the findings among a purposive sample of parents from urban GP practices in the United Kingdom [20]. Parents in Australia, Canada, the UK, the Netherlands and Iceland [21] all seem aware of potential harmful effects of antibiotics, especially when used repeatedly.

Other qualitative studies of parents' perceptions on symptom management have focused on acutely ill children or those with respiratory tract infections [23-31]. In line with our findings, these studies show that parents mainly consult their GP for diagnosis and for reassurance, and they do not want antibiotics when they will not relieve current symptoms.

The studies on parents' views of AOM management confirm parents' difficulties in recognising AOM as the underlying cause of their children's distress, and their search for reassurance, as well as ways to relieve their child's symptoms [5] [20, 21]. In general, parents value a discussion of management options by their GP [5] [20,21].

Our study shows that parents generally seem to prefer a non-antibiotic prescribing strategy and accept pain medication as standalone therapy where possible. On the other hand, the GPs in our trial that were interviewed in a separate qualitative study tend to focus on treating the infection and whether or not to prescribe antibiotics for AOM [32]. This prescribing behaviour often results from perceived parental pressure or feelings of helplessness in managing symptoms with analgesics. Importantly, both parents and GPs are aware of the pain caused by of AOM, and strive to relieve the child's symptoms. We believe there is a need for research identifying strategies on how to alleviate these symptoms most effectively, and recommend GPs to address pain management in AOM consultations.

\section{Strengths \& limitations}

Regular review and discussion of coding and analysis with all members of a multidisciplinary research team (researcher triangulation) has contributed to consistency in data synthesis and interpretation [33-35], as well as increased trustworthiness [36]. The different background of coders (sociologist and physician) enhances reliability and reflexivity and has helped to set aside any preconceptions [37].

One could argue that participants changed their behaviour due to trial participation, the so-called Hawthorne-effect, 
and that this may have influenced parents' perceptions on AOM management. We aimed to minimise this issue by encouraging parents to provide their views and expectations regardless of any study procedures, and by including parents from the control group who were not aware of the intervention and received usual care.

Our study population only included parents who consulted their GP because their child suffered from AOM symptoms. As such, it is uncertain to what extent our findings can be transferred to the substantial number of parents who self-manage their child's AOM episodes at home [38].

Campaigns and guidelines in the last decades in the Netherlands have emphasized the importance of adequate analgesia and judicious antibiotic use for upper respiratory tract infections in children, and antibiotic prescribing rates in general practice are among the lowest in the world [39]. Parents in our sample generally seem aware of this antibiotic policy, but not of the full range of analgesic possibilities. Our results may be transferrable to countries with comparable attitudes towards AOM management, regardless of who is primarily responsible for managing childhood AOM episodes (e.g. GP, primary care paediatrician, or nurse practitioner), under the condition that all healthcare professionals consistently provide parents with management options, that are in line with public campaigns and/or clinical practice guidelines $[40,41]$.

\section{Conclusion}

In conclusion, parents of children with AOM consult their GP to help cope with uncertainties in recognising $\mathrm{AOM}$ and to receive management advice. It is important that GPs, and other clinicians involved in the care of children with AOM, are aware of parents' lack of understanding of the role of pain medication in managing $\mathrm{AOM}$ and address this during the consultation, to better align with parents' views and expectations.

\section{Additional files}

Additional file 1: Translated parent information leaflet about pain relief for children with middle ear infection. To be used by the general practitioner as part of the intervention, to discuss pain management in each acute otitis media consultation (PDF 666 kb)

Additional file 2: Interview guide as used by the study physician as guidance for the semi-structured interviews. (DOCX 28 kb)

\section{Abbreviations}

AOM: Acute otitis media; GP : General practitioner; OTC: Over-the-counter

\section{Acknowledgements}

The authors would like to thank all parents who participated in this study. We particularly thank Liz Gorin for her professional assistance in quote and topic list translation, and Anik Patel, MD, for reviewing grammar and spelling.

\section{Funding}

This qualitative study was performed alongside a cluster randomized controlled trial $(C R C T)$ aimed at optimizing pain management in children with acute otitis media. The CRCT was supported by a grant from the Netherlands Organization for Health Research and Development (ZonMw) - HGOG subprogram (project number 839110005$)$. The funders had no role in study design, data collection and analysis, decision to publish, or preparation of this work.

\section{Availability of data and materials}

The datasets used and analyzed during the current study are available from the corresponding author on reasonable request.

\section{Authors' contributions}

RTVU and RPV drafted the first version of the manuscript. AGMS, RAMJD and SA provided feedback on the manuscript. All authors approved the final version of the manuscript.

\section{Ethics approval and consent to participate}

The Medical Ethics Review Committee of the University Medical Center Utrecht, the Netherlands, reviewed the study protocol (protocol 17-801/C) and confirmed that formal approval was not required, since the Medical Research Involving Human Subjects Act (WMO) does not apply.

Participation was voluntary, and only after written informed consent. Participants were informed that the interviews would be tape-recorded and data would be treated anonymously, confidentially and stored securely in accordance with data protection legislation. Interview tapes and transcripts were coded to protect interviewee anonymity and access to codes was restricted to the interviewers.

\section{Consent for publication}

Not applicable.

\section{Competing interests}

None of the authors have relationships with companies that might have an interest in the submitted work; neither do they have non-financial interests that may be relevant to the submitted work.

\section{Publisher's Note}

Springer Nature remains neutral with regard to jurisdictional claims in published maps and institutional affiliations.

\section{Author details}

${ }^{1}$ Julius Center for Health Sciences and Primary Care, University Medical Center Utrecht, University Utrecht, Office number FAC 5.09, P.O. Box 85500, 3508, GA, Utrecht, The Netherlands. 2evidENT, Ear Institute, University College London, London, UK. ${ }^{3}$ Department of Primary Care and Interdisciplinary Care, University of Antwerp, Antwerp, Belgium.

Received: 24 October 2018 Accepted: 15 January 2019

Published online: 23 January 2019

\section{References}

1. McGrath LJ, Becker-Dreps S, Pate V, Brookhart MA. Trends in antibiotic treatment of acute otitis media and treatment failure in children, 2000-2011. PLoS One. 2013;8(12):e81210.

2. Tyrstrup M, van der Velden A, Engstrom S, Goderis G, Molstad S, Verheij T, et al. Antibiotic prescribing in relation to diagnoses and consultation rates in Belgium, the Netherlands and Sweden: use of European quality indicators. Scand J Prim Health Care. 2017;35(1):10-8.

3. Marom T, Tan A, Wilkinson GS, Pierson KS, Freeman JL, Chonmaitree T. Trends in otitis media-related health care use in the United States, 20012011. JAMA Pediatr. 2014;168(1):68-75.

4. Barber $\mathrm{C}$, Ille $\mathrm{S}$, Vergison A, Coates $\mathrm{H}$. Acute otitis media in young children what do parents say? Int J Pediatr Otorhinolaryngol. 2014;78:300-6.

5. Hansen MP, Howlett J, Del Mar C, Hoffmann TC. Parents' beliefs and knowledge about the management of acute otitis media: A qualitative study. BMC Fam Pract. 2015;16:82.

6. Lieberthal AS, Carroll AE, Chonmaitree T, Ganiats TG, Hoberman A, Jackson MA, et al. Clinical practice guideline: the diagnosis and Management of Acute Otitis Media. Pediatrics. 2013;131:e964-99. 
7. NICE. Otitis media (acute): antimicrobial prescribing (NG91). NICE; 2018 Available from: nice.org.uk/guidance/ng91.

8. Coco A, Vernacchio L, Horst M, Anderson A. Management of Acute Otitis Media after Publication of the 2004 AAP and AAFP clinical practice guideline. Pediatrics. 2010;125:214-20.

9. Tähtinen PA, Boonacker CWB, Rovers MM, Schilder AGM, Huovinen P Liuksila PR, et al. Parental experiences and attitudes regarding the management of acute otitis media - a comparative questionnaire between Finland and the Netherlands. Fam Pract. 2009;26:488-92.

10. Twycross AM, Williams AM, Sunderland R. Parental attitudes to children's pain and analgesic drugs in the United Kingdom. J Child Heal Care. 2015; 19(3):402-11.

11. Li SF, Lacher B, Crain EF. Acetaminophen and ibuprofen dosing by parents. Pediatr Emerg Care. 2000;16(6):394-7.

12. Boonacker CWB, Hoes AW, Dikhoff M-J, Schilder AGM, Rovers MM. Interventions in health care professionals to improve treatment in children with upper respiratory tract infections. Int J Pediatr Otorhinolaryngol. 2010; 74:1113-21.

13. Tonkin-Crine SKG, Tan PS, van Hecke O, Wang K, Roberts NW, McCullough $A$, et al. Clinician-targeted interventions to influence antibiotic prescribing behaviour for acute respiratory infections in primary care: an overview of systematic reviews (review). Cochrane Database Syst Rev. 2017;9:CD012252.

14. Damoiseaux RA, Venekamp RP, Eekhof J, Bennebroek Gravenhorst F, Schoch A, Burgers J, et al. M09 NHG guideline acute otitis media in children. Huisarts Wet. 2014;57(12):648

15. Tomlinson D, von Baeyer C, Stinson J, Sung L. A systematic review of faces scales for the self-report of pain intensity in children. Pediatrics. 2010;126:e1168-98.

16. van Uum RT, Venekamp RP, Sjoukes A, van der Pol AC, de Wit AG, Schilder $A G$, et al. Optimising pain management in children with acute otitis media through a primary care-based multifaceted educational intervention: study protocol for a cluster randomised controlled trial. Trials. 2018;19(501):1-9.

17. Halcomb EJ, Furler JS, Hermiz OS, Blackberry ID, Smith JP, Richmond RL, et al. Process evaluation of a practice nurse-led smoking cessation trial in Australian general practice: views of general practitioners and practice nurses. Fam Pract. 2015;32(4):468-73.

18. Braun V, Clarke V. Using thematic analysis in psychology. Qual Res Psychol. 2006;3(2):77-101.

19. Ritchie J, Spencer L. In: Bryman A, Burgess R, editors. Qualitative data analysis for applied policy research. London: Routledge; 1993. p. 173-94.

20. Russell D, Luthra M, Wright J, Golby M, Plastow L, Marshall M. A qualitative investigation of parents' concerns, experiences and expectations in managing otitis media in children: implications for general practitioners. Prim Heal Care Res Dev. 2003;4:85-93.

21. Jónsson $H$, Haraldsson RH. Parents' perspectives on otitis media and antibiotics: a qualitative study. Scand J Prim Health Care. 2002;20:35-9.

22. Meherali S, Campbell A, Hartling L, Scott S. Understanding parents' experiences and information needs on pediatric acute otitis media: a qualitative study. J Patient Exp. 2018:1-9. https://doi.org/10.1177/ 2374373518771362

23. McDermott L, Leydon GM, Halls A, Kelly J, Nagle A, White J, et al. Qualitative interview study of antibiotics and self-management strategies for respiratory infections in primary care. BMJ Open. 2017;7(11):e016903.

24. Kai J. What worries parents when their preschool children are acutely ill, and why: a qualitative study. BMJ. 1996;313:983-6.

25. Lucas PJ, Cabral C, Hay AD, Horwood J. A systematic review of parent and clinician views and perceptions that influence prescribing decisions in relation to acute childhood infections in primary care. Scand J Prim Health Care. 2015;33(1):11-20.

26. Dekker ARJ, De Groot E, Sebalj T, Yardley L, Cals JWL, Verheij TJM. Parents' attitudes and views regarding antibiotics in the management of respiratory tract infections in children : a qualitative study of the influence of an information booklet. BJGP Open. 2018;2:1-9.

27. Halls A, Van't Hoff C, Little P, Verheij T, Leydon GM. Qualitative interview study of parents' perspectives, concerns and experiences of the management of lower respiratory tract infections in children in primary care. BMJ Open. 2017;7:e015701.

28. Coxeter PD, Del Mar C, Hoffmann TC. Parents' expectations and experiences of antibiotics for acute respiratory infections in primary care. Ann Fam Med. 2017;15(2):149-54.
29. Cabral C, Ingram J, Lucas PJ, Redmond NM, Kai J, Hay AD, et al. Influence of clinical communication on parents' antibiotic expectations for children with respiratory tract infections. Ann Fam Med. 2016;14(2):141-7.

30. Ingram J, Cabral C, Hay AD, Lucas PJ, Horwood J. Parents' information needs, self-efficacy and influences on consulting for childhood respiratory tract infections: A qualitative study. BMC Fam Pract. 2013;14:106.

31. Cabral C, Lucas PJ, Ingram J, Hay AD, Horwood J. "It's safer to..." parent consulting and clinician antibiotic prescribing decisions for children with respiratory tract infections: an analysis across four qualitative studies. Soc Sci Med. 2015;136-137:156-64.

32. van Uum RT, Sjoukes A, Venekamp RP, Schilder AG, de Groot E, Damoiseaux $R A$, et al. Pain management in acute otitis media: an exploration of general practitioners' views and expectations alongside a trial of an educational intervention. BJGP Open. 2018. https://doi.org/10.3399/bjgpopen18X101620.

33. Patton $\mathrm{MQ}$. Enhancing the quality and credibility of qualitative analysis. Health Serv Res. 1999;34(5):1189-208.

34. Pope C, Ziebland S, Mays N. Qualitative research in health care Analysing qualitative data. BMJ. 2000;320:114-6.

35. Seale C. Quality in qualitative research. Qual Inq. 1999;5(4):465-78.

36. Korstjens I, Moser A. Series: Practical guidance to qualitative research. Part 4: Trustworthiness and publishing. Eur J Gen Pract. 2017;24:120-4.

37. Malterud K. Qualitative research: standards, challenges, and guidelines. Lancet. 2001;358:483-8.

38. Fortanier AC, Venekamp RP, de Hoog MLA, Uiterwaal CSPM, van der Gugten AC, van der Ent CK, et al. Parent-Reported Symptoms of Acute Otitis Media during the First Year of Life: What Is beneath the Surface? PLoS One. 2015; 10(4):e0121572 Available from: http://dx.plos.org/10.1371/journal.pone.0121572.

39. van den Broek d'Obrenan J, TJM V, Numans ME, van der Velden AW. Antibiotic use in Dutch primary care: relation between diagnosis, consultation and treatment. J Antimicrob Chemother. 2014;69:1701-7.

40. Strandberg EL, Brorsson A, André M, Gröndal H, Mölstad S, Hedin K. Interacting factors associated with Low antibiotic prescribing for respiratory tract infections in primary health care - A mixed methods study in Sweden. BMC Fam Pract. 2016;17:78 Available from: https://doi.org/10.1186/s12875016-0494-z.

41. Filippini M, Ortiz LGG, Masiero G. Assessing the impact of national antibiotic campaigns in Europe. Eur J Health Econ. 2013:14(4):587-99.

\section{Ready to submit your research? Choose BMC and benefit from:}

- fast, convenient online submission

- thorough peer review by experienced researchers in your field

- rapid publication on acceptance

- support for research data, including large and complex data types

- gold Open Access which fosters wider collaboration and increased citations

- maximum visibility for your research: over $100 \mathrm{M}$ website views per year

At $B M C$, research is always in progress.

Learn more biomedcentral.com/submissions 